\title{
Materias primas no tradicionales en la industria cerámica
}

\author{
R. CALIGARIS', N. QUARANTA², M. CALIGARIS Y E. BENAVIDEZ \\ Facultad Regional San Nicolás, Universidad Tecnológica Nacional, 2900 San Nicolás, Argentina \\ ${ }^{1}$ Investigador Científico del Consejo Nacional de Investigaciones Científicas y Técnicas. CONICET. \\ ${ }^{2}$ Investigador Científico de la Comisión de Investigaciones Científicas. CIC.
}

\begin{abstract}
La incorporación de materiales de desecho en el ciclo productivo es una alternativa de interés en la industria moderna que conduce tanto a un beneficio económico como a una considerable reducción del impacto sobre el medio ambiente. En este trabajo se comparan estudios realizados sobre la reutilización de diversos residuos: vidrio, aisladores eléctricos y cenizas volantes. Estos materiales fueron molidos y clasificados por tamaño de partículas para ser usados en la preparación de muestras representativas. Las diferentes etapas de tratamiento se establecieron según los procesos habituales de producción de la industria cerámica. Algunos de los polvos obtenidos se utilizaron sin aditivos, mientras que otros fueron mezclados en distintas proporciones con diversos componentes cerámicos con el fin de mejorar sus propiedades finales. Todos los compactos, obtenidos por prensado uniaxial de estos polvos, recibieron tratamientos térmicos adecuados a sus composiciones, para luego ser caracterizados por diversas técnicas entre las que se pueden mencionar: dilatometría, difracción de rayos X, porosidad, microdureza, microscopías óptica y electrónica de barrido y análisis por dispersión de energías. Del análisis de los resultados obtenidos en estos estudios, se concluyó que los materiales ensayados constituyen una potencial fuente de materia prima para la industria cerámica.
\end{abstract}

Palabras clave: materiales cerámicos, materias primas, reciclado, residuos, densificación.

\section{Non traditional raw material in ceramic industry}

The wastes incorporation to the production cycle is an interesting alternative in modern industry. This gives rise to not only an economical profit but to a considerable decrease in environmental impact. Studies made about recycling of several wastes: glass, electric insulators and fly ash are compared in this work. The materials were crushed and separated by particle size distribution in order to prepare representative samples. Different treatment steps were carried out as is usual in the process of ceramic industries. Some of the obtained powders were used without additives while other were mixed in different ratios with other ceramic components in order to improve their final properties. All the compacts, obtained by uniaxial pressing of the powders, received thermal treatments which were adequate to their compositions. They were then characterised by several techniques including dilatometry, $X$ ray diffraction, porosity, microhardness, optical and scanning electron microscopy and energy dispersion analysis (SEM/EDX). From this investigation it was concluded that the studied materials constitute a potential source of raw materials for the ceramic industry.

Key words: ceramic materials, raw material, recycling, wastes, densification.

\section{INTRODUCCIÓN}

Como productos secundarios en los procesos industriales y como consecuencia de las actividades humanas se genera una gran cantidad de residuos, cuyo control o tratamiento constituye uno de los problemas más importantes en la actualidad, principalmente en los países desarrollados. La dificultad de establecer lugares adecuados para el depósito de aquellos residuos que sólo generan contaminación volumétrica ha provocado que se contemple como solución alternativa el reciclado de los mismos, evitando no solamente el impacto que su acumulación produce sino los costos que su traslado y almacenamiento originan.

En los últimos años se han realizado numerosas investigaciones que emplean diferentes técnicas de tratamientos de residuos y desarrollan nuevas tecnologías, con el objetivo de reducir el impacto que tales desechos industriales ocasionan en el entorno (1-5). En este trabajo se analiza la reutilización de diversos residuos como materia prima no tradicional de la industria cerámica: vidrio, aisladores eléctricos y cenizas volantes.

El desecho de vidrio no agrede en realidad al ambiente, ya que las materias primas con las que éstos se fabrican se utilizan en la misma proporción en la que se encuentran en la corteza terrestre. En este trabajo, el vidrio de rezago se utiliza como componente principal para la obtención de materiales cerámicos. Se estudian además, distintas mezclas con otros materiales de tipo silicoaluminoso y alúmina.

Los aisladores cerámicos utilizados por las empresas eléctricas quedan en desuso, produciendo serios problemas de contaminación volumétrica. Se estudiaron dos clases de aisladores eléctricos de porcelana de distinta procedencia comercial. 
Los polvos obtenidos a partir de estos materiales están constituídos fundamentalmente por mullita, pero en un caso son ricos en alúmina y en el otro tienen una importante cantidad de cuarzo.

Las cenizas volantes son un subproducto común en las centrales termoeléctricas. Actualmente un pequeño porcentaje se utiliza en la industria del cemento y la porción restante como relleno de suelos, lo que no resulta satisfactorio ni desde el punto de vista económico ni ambiental (6). Los componentes principales de las cenizas son: sílice, alúmina, óxido de hierro y óxido de calcio.

En el presente estudio se muestra que es posible obtener piezas compactas con un alto contenido de mullita, a partir de un adecuado tratamiento de los residuos estudiados, haciendo posible su utilización como materia prima en los procesos productivos de la industria cerámica, sin necesidad de modificación de los mismos.

\section{PARTE EXPERIMENTAL}

\subsection{Preparación de las muestras}

\subsubsection{VIDRIOS}

Se utilizaron vidrios verdes residuales que fueron molidos y posteriormente seleccionados según su distribución granulométrica. Estos polvos se mezclaron con otros materiales y en diferentes proporciones. Los materiales utilizados fueron: minerales naturales como bentonita y silimanita, y alúmina comercial (Aldrich, pureza 99,8\%, tamaño de partícula $<10 \mu \mathrm{m}$ ). También se ensayaron muestras de vidrio sin aditivos con fines comparativos.

Las muestras vidrio-bentonita fueron preparadas con diferentes proporciones de bentonita entre 5 y $15 \%$ en peso. Las composiciones de ambos materiales se muestran en la Tabla I.

TABLA I COMPOSICIÓN PORCENTUAL EN PESO DE ÓXIDOS EN EL VIDRIO Y LA BENTONITA.

\begin{tabular}{|c|c|c|c|c|c|c|c|c|}
\hline & $\mathrm{Na}_{2} \mathbf{O}$ & $\mathrm{MgO}$ & $\mathrm{Al}_{2} \mathbf{O}_{3}$ & $\mathrm{SiO}_{2}$ & $\mathbf{K}_{2} \mathbf{O}$ & $\mathbf{C a O}$ & $\mathrm{TiO}_{2}$ & $\mathbf{F e}_{2} \mathbf{O}_{3}$ \\
\hline Vidrio & 4,0 & 1,3 & 10,0 & 70,5 & 1,8 & 8,3 & --- & 4,1 \\
\hline Bentonita & --- & 0,5 & 25,0 & 51,4 & 1,0 & 0,9 & 1,0 & 20,2 \\
\hline
\end{tabular}

Los compactos obtenidos a partir de estas mezclas se sometieron a tratamientos térmicos en el rango: $550^{\circ} \mathrm{C}-750^{\circ} \mathrm{C}$, para determinar la temperatura de sinterizado (Ts) y la temperatura de vitrificación (Tv). Ambas fueron determinadas por observaciones de microscopía óptica en muestras calcinadas con pasos de $25^{\circ} \mathrm{C}(7)$.

Un procedimiento similar fue utilizado en el estudio del sistema vidrio-silimanita. El porcentaje de silimanita adicionado varió entre el $20 \%$ y el $45 \%$. El intervalo de temperaturas estudiado estuvo entre los $600-900^{\circ} \mathrm{C}$ (8). Previo a su utilización, el mineral de silimanita, tal como se extrae de su fuente natural, fue sometido a un tratamiento con el fin de disminuir ciertas impurezas como $\mathrm{Fe}_{2} \mathrm{O}_{3}$ y mica, que provocan inconvenientes en el proceso de sinterización de las muestras (9).

En las muestras obtenidas con adición de $\mathrm{Al}_{2} \mathrm{O}_{3}$, los porcentajes utilizados fueron los adecuados para alcanzar la relación estequiométrica correspondiente a la mullita $\left(3 \mathrm{Al}_{2} \mathrm{O}_{3} \cdot 2 \mathrm{SiO}_{2}\right)$.
Los compactos de vidrio-alúmina fueron tratados hasta sinterización a $750^{\circ} \mathrm{C}$ y luego con temperaturas hasta $1550^{\circ} \mathrm{C}(10)$.

\subsubsection{AISLADORES ELECTRICOS}

Se utilizaron dos tipos de aisladores eléctricos de diferentes orígenes comerciales, que fueron identificados como $E G$ y $E M$. La parte cerámica de los mismos fue llevada al estado de polvo en un molino de disco oscilante. Los polvos así obtenidos presentaron las siguientes fases cristalográficas:

- EG: cuarzo, mullita y alúmina.

- EM: cuarzo, mullita, tridimita y súlice cúbica.

La relación entre las intensidades de los picos mullita/cuarzo posee un valor similar en ambas muestras, indicando que la proporción entre estas fases es parecida.

A partir de los polvos obtenidos se conformaron probetas de cada tipo, EM y EG (11). Además se prepararon muestras con diferentes proporciones de ambos (12).

Los tratamientos de sinterizado se establecieron entre los $1200^{\circ} \mathrm{C}$ y $\operatorname{los} 1650^{\circ} \mathrm{C}$.

\subsubsection{CENIZAS VOLANTES}

Las cenizas provenientes de una planta generadora de energía eléctrica varían su composición de acuerdo al tipo de combustible del que provienen. Esto genera dificultades al momento de establecer parámetros de tratamiento. En este estudio se tomaron muestras con características extremas en cuanto al porcentaje de carbón no quemado que contienen. Así, una de las cenizas utilizadas (denominada $C 1$ ) posee una pérdida del $18 \%$ de peso de carbón cuando se calienta a $750^{\circ} \mathrm{C}$, y la otra ceniza seleccionada (denominada C2) pierde alrededor del $13 \%$ cuando se somete a este tratamiento térmico. En la Tabla II se listan los porcentajes de óxidos presentes en ambas muestras de cenizas.

TABLA II: COMPOSICIÓN DE LAS CENIZAS.

\begin{tabular}{|c|c|c|c|c|c|c|c|}
\hline Muestra & $\mathbf{M g O}$ & $\mathrm{Al}_{2} \mathbf{O}_{3}$ & $\mathbf{S i O}_{2}$ & $\mathbf{K}_{2} \mathbf{O}$ & $\mathbf{C a O}$ & $\mathbf{T i O}_{2}$ & $\mathbf{F e}_{2} \mathbf{O}_{3}$ \\
\hline $\mathbf{C} 1$ & 0,8 & 22,1 & 59,7 & 1,4 & 5,5 & 2,1 & 8,5 \\
\hline $\mathbf{C} 2$ & 0,6 & 24,5 & 58,4 & 1,6 & 5,1 & 2,4 & 7,5 \\
\hline
\end{tabular}

Las cenizas volantes, tal como se originan en el proceso industrial, sin tratamientos previos $y$ sin aditivos fueron utilizadas para obtener compactos que se trataron a temperaturas entre los $700-1300^{\circ} \mathrm{C}$ (13). Por otro lado, se prepararon otras muestras con $\boldsymbol{C} \mathbf{1}$ adicionándoles porcentajes de hasta el $40 \%$ de $\mathrm{Al}_{2} \mathrm{O}_{3}$, que fueron tratadas en el rango de los $1200-1600^{\circ} \mathrm{C}(14)$.

\subsection{Caracterización de las muestras}

La caracterización de las muestras se llevó a cabo utilizando los métodos habituales para el ensayo de materiales cerámicos. La microestructura se estudió por microscopía óptica de las superficies pulidas de muestras seleccionadas, con un microscopio marca Zeiss, con cámara Philips y digitalizador de imágenes. Se completó el estudio con análisis por micros- 
copía electrónica de barrido y análisis por dispersión electrónica de rayos X (EDX). Se utilizó un equipo Philips 505. La porosidad de las muestras se determinó por el método de Arquímedes. Los análisis de difracción de rayos X llevados a cabo para determinar las fases cristalinas presentes en los materiales, se realizaron con un equipo Philips PW 1390. Se determinaron los valores de microdureza Vickers con un equipo marca Shimatzu 2000, utilizando una carga de $300 \mathrm{~g}$ durante 15 segundos. Los mecanismos de densificación de los compactos producidos fueron estudiados mediante dilatometría en un dilatómetro Dilatronic Theta.

\section{RESULTADOS Y DISCUSION}

\subsection{Vidrios}

En los compactos obtenidos a partir de vidrio verde y bentonita, las temperaturas de sinterización y vitrificación determinadas fueron mayores con el correspondiente aumento del contenido de bentonita, resultando por ejemplo, para las muestras con $15 \%$, Tsint.: $675^{\circ} \mathrm{C}$ y Tvit.: $710^{\circ} \mathrm{C}$. La contracción de las muestras resultó similar independientemente de la granulometría utilizada, pero con tendencia a disminuir a mayores contenidos del aglomerante. El análisis por rayos $\mathrm{X}$ mostró la presencia de fases amorfas en todos los compactos estudiados. Los materiales sinterizados presentaron uniones entre granos, mientras que los vitrificados mostraron una fase compacta con escasa presencia de poros. Las medidas de microdureza Vickers presentaron valores similares, $\mathrm{Hv} \sim 500 \mathrm{Kgf} / \mathrm{mm}^{2}$ (5000 MPa) independientemente del contenido de bentonita. Sin embargo, las muestras con $15 \%$ de bentonita resultaron más frágiles. De todos los resultados obtenidos se desprende que los compactos con $10 \%$ de bentonita presentaron las propiedades más adecuadas para el uso deseado.

En las muestras vidrio-silimanita se determinó que $750^{\circ} \mathrm{C}$ es la temperatura óptima de sinterización. Los análisis de porosidad y densidad mostraron en las probetas con mayor contenido de silimanita una porosidad y absorción de agua superiores. La observación por microscopía reveló que se produjeron uniones entre granos de silimanita y vidrio, incrementándose el número de éstas con el porcentaje de silimanita. Esto le confiere al material propiedades mecánicas adecuadas para su uso como material cerámico.

En el análisis de las muestras vidrio-alúmina se determinó que hasta $1250^{\circ} \mathrm{C}$ no se advirtieron cambios microestructurales importantes. La observación por microscopía reveló independientemente la fase vítrea original y la alúmina agregada, y algunos silicoaluminatos comenzando a desarrollarse en la zona superficial de las probetas. A $1300^{\circ} \mathrm{C}$ se obtuvieron estructuras densas sin zonas fundidas. A temperaturas mayores se observaron zonas de vidrio fundido y presencia de granos de alúmina en los bordes de los poros, inmersos en una matriz silicoaluminosa. A $1500^{\circ} \mathrm{C}$ se observó pérdida de aristas y principio de burbujeo. Con el aumento de temperatura de tratamiento se produjo un incremento considerable de la porosidad y la microdureza de los compactos. Los análisis por difracción de rayos $X$ revelaron presencia de $\alpha$-alúmina y numerosos picos pequeños correspondientes a silicatos de aluminio y calcio. La temperatura de tratamiento más adecuada para producir compactos densos, que mantienen bordes bien definidos resultó $1300^{\circ} \mathrm{C}$.

\subsection{Aisladores eléctricos}

En el análisis de los espectros de difracción de rayos $\mathrm{X}$ de la muestra EM se observó una evolución de las fases presentes a medida que aumenta la temperatura, con disminución de los picos de cuarzo, tridimita y sílice cúbica, a expensas de la formación de un fondo característico de una fase amorfa. En las muestras EG, estos análisis revelaron una importante formación de mullita, resultando a $1400^{\circ} \mathrm{C}$ las fases mullita y alúmina las únicas detectables. A la temperatura de $1600^{\circ} \mathrm{C}$ se observó deformación de las muestras prensadas. Las curvas dilatométricas de los materiales obtenidos concuerdan con los datos de porosidad y densidad. A partir de ambos estudios se puede determinar que la región entre los $1250^{\circ} \mathrm{C}$ y $1300^{\circ} \mathrm{C}$ es la propicia para generar materiales cerámicos más densos.

Las muestras producidas con la mezcla de los polvos de los dos tipos de aisladores, presentaron características de densificación intermedias. Su evolución microestructural se ha observado por microscopía óptica. Las muestras tratadas a $1200^{\circ} \mathrm{C}$ contienen una gran cantidad de poros, que disminuyeron en número pero incrementaron su tamaño con el aumento de la temperatura de tratamiento. En todas las muestras se observaron bastones de alúmina libre que disminuyeron en fracción de volumen a medida que se elevó la temperatura de tratamiento, ya que reaccionaron con la sílice libre para formar mullita. Los valores de microdureza de las distintas probetas presentaron poca variación, aunque valores algo superiores se obtuvieron en las muestras que poseen un porcentaje de alúmina superior (por su mayor contenido de $E G$ ). En el rango $1250^{\circ} \mathrm{C}-1300^{\circ} \mathrm{C}$ se obtuvieron las mayores densidades. Desde el punto de vista de la factibilidad de reprocesamiento de estos aisladores resulta beneficioso, debido a que son éstas las temperaturas normalmente utilizadas en la industria de los cerámicos.

\subsection{Cenizas volantes}

Las probetas prensadas de las cenizas $\mathrm{C} 1$ y $\mathrm{C} 2$, iniciaron su etapa de sinterización a $1100^{\circ} \mathrm{C}$, presentando apariencia compacta con bordes bien definidos. Con el aumento de la temperatura, entre 1100 y $1260^{\circ} \mathrm{C}$, los valores de microdureza Vickers resultaron cada vez mayores. A partir de $1260^{\circ} \mathrm{C}$ aumentó la porosidad de las muestras, el vidriado superficial y el curvado de las aristas. Los diagramas de rayos $\mathrm{X}$ de las cenizas originales, antes del tratamiento térmico, mostraron presencia de cuarzo y algunos picos de mullita. A la temperatura de sinterización $\left(1100^{\circ} \mathrm{C}\right)$, se completó el diagrama de mullita, disminuyó la presencia de cuarzo, y aparecieron picos de anortita. Desde $1100^{\circ} \mathrm{C}$ hasta $1300^{\circ} \mathrm{C}$, la estructura evolucionó de manera que los únicos picos presentes fueron los de la fase mullita. La temperatura óptima para el tratamiento de las cenizas fue $1200^{\circ} \mathrm{C}$.

Las muestras obtenidas a partir de las mezclas ceniza-alúmina presentaron porosidades prácticamente constantes hasta $1400^{\circ} \mathrm{C}$. Por encima de esta temperatura se observó inicio de burbujeo en las probetas con 10 y $20 \%$ de alúmina, mientras que los que poseen porcentajes superiores no sufrieron deformación aún cuando la temperatura superó los $1550^{\circ} \mathrm{C}$. Los valores de microdureza están en relación directa con la formación de mullita y con la cantidad de alúmina presente. Los diagramas de difracción de rayos $\mathrm{X}$ de las muestras con mayores adiciones de alúmina presentaron picos correspondientes a mullita y $\alpha$-alúmina sin reaccionar. Se observaron a elevados aumentos bastones de alúmina y cristales de mullita en una matriz de sílice, alúmina y cal, con bajo contenido de 
Fe y Ti, según los análisis SEM-EDX (figura 1). Los materiales cerámicos con mejores propiedades se obtuvieron por la adición del $30 \%$ de alúmina a las cenizas y con un tratamiento térmico a $1500^{\circ} \mathrm{C}$.

\section{CONCLUSIONES}

Los resultados de los estudios realizados en este trabajo permiten concluir que es posible la incorporación de un alto porcentaje de los materiales residuales estudiados, en los procesos de producción de la industria cerámica. Sumado al beneficio ambiental que implica el reciclado de estos materiales, existe un potencial beneficio económico en la sustitución de la materia prima tradicional. Este cambio en los materiales de partida no requiere de una modificación de los procesos de producción actualmente utilizados, ya que las temperaturas de tratamiento determinadas para obtener las propiedades óptimas son las habitualmente empleadas en la industria.

\section{AGRADECIMIENTOS}

Los autores agradecen el apoyo recibido de CONICET y CIC, a través de los subsidios otorgados, así como la colaboración brindada por la Empresa Central Térmica San Nicolás S.A.

\section{BIBLIOGRAFÍA}

1. R. Caligaris, L. Zamboni, C. Ortiz and M. Caligaris. “Bricks from coal tailings" Tail \& Bricks Int. 6 41-42 (1990).

2. J. Bennett, K. Kwong and S.W.Sikich. "Recycling and Disposal of Refractories". Am.Ceram.Society Bulletin, 7412 71-77 (1995).

3. Boccaccini, M. Bücker, J. Bossert and K. Marszdek. "Glass Matrix Composites from coal flyash and waste glass". Waste Management, 171 39-45 (1997).

4. L. Barbieri, T. Manfredini, I. Queralt, J. Rincón and M. Romero. “Vitrification of flyash from thermal power stations". Glass Technol., 38 5 $165-170$ (1997).

5. M. Romero and J. M. Rincón. "Preparation and properties of high iron oxide content glasses obtained from industrial wastes". J. European Ceram. Society, 18 153-160 (1998).

6. J. R. Conner. "Portland Cement/Flyash Processes". 417-426 en Chemical Fixation and Solidification of Hazardous Wastes. Editorial Van Nostrand Reinhold. New York. (1990).

7. M. Caligaris, S. Camelli, L. Zamboni, N. Quaranta et R. Caligaris. “Utilisation de verre recyclé pour la production de patês céramiques". L'industrie céramique \& verrière, 926 [5] 338-341 (1997).

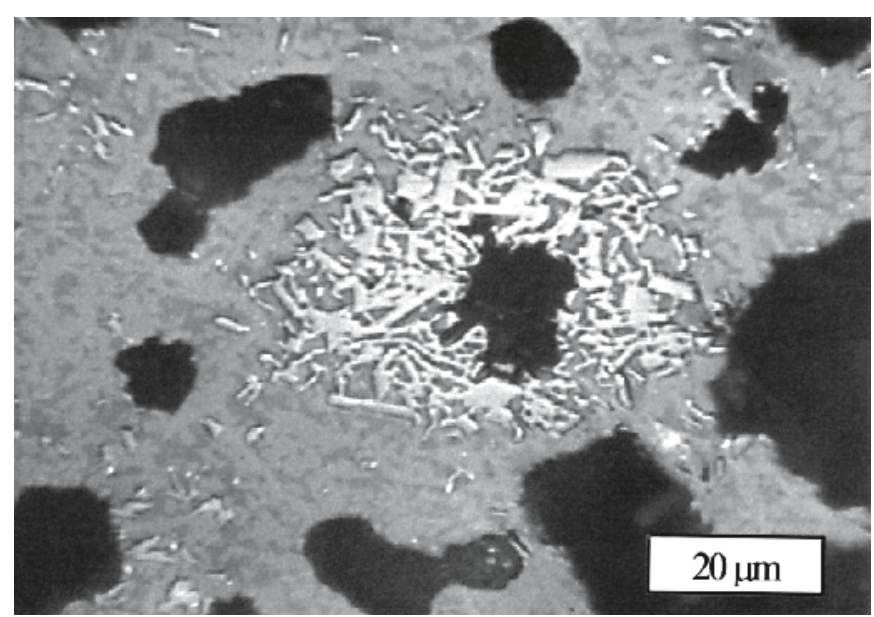

Figura 1: Microestructura de la muestra C1 con 40\% de alúmina.

8. S. Camelli, L. Zamboni, M. Caligaris, N. Quaranta y R. Caligaris. “Mezclas silimanita-vidrio para uso cerámico". Anales de Jornadas de la Sociedad Argentina de Materiales (SAM 97), Tandil (Argentina), Mayo 1997.

9. J. Geisler, L. Zamboni, N. Quaranta y R. Caligaris. “Mullite da reazione termica di sillimanite naturale con aggiunta di $\mathrm{SiC}^{\prime}$. Ceramurgia año XXVI [6] 362-365 (1996)

10. N. Quaranta, M. Caligaris, S. Camelli, L. Zamboni y R. Caligaris. "Piezas cerámicas a partir de vidrio de descarte con adición de alúmina". Actas del IX Congreso Internacional y III del Mercosur de Cerámica, Vidrio y Refractarios, Olavarría (Argentina), Mayo 1998.

11. N. Quaranta, M. Caligaris and E.Benavidez. "Recycling Electric Insulators as a Way to have Mullite". $3^{\circ}$ International Meeting of Pacific Rim Ceramic Societies. PacRim 3. Kyongju, (Corea), Septiembre 1998.

12. E. Benavidez, M. Caligaris, N. Quaranta and A. Boccaccini. "Electric Companies Wastes as Ceramic Raw Material". Sixth Conference and Exhibition of the European Ceramic Society (6 E.Cer.S'99). Brighton, (Inglaterra), Junio 1999.

13. N. Quaranta, S. Camelli, M. Caligaris y R. Caligaris. “Cenizas Industriales: Utilización en Materiales Cerámicos" 2nd International Congress on Metallurgy and Materials Technology. San Pablo, (Brasil), Octubre 1997.

14. N. Quaranta, S. Camelli, M. Caligaris, E. Benavidez, R. Caligaris, A. Boccaccini and H. Kern. "Fabrication of mullite ceramics using fly ash and alumina". EUROMAT'99. Munich, (Alemania), Septiembre 1999.

Recibido: 19-10-99

Aceptado: 2-5-00 